Canadian

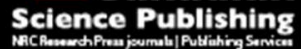

Canadian Journal of Physiology and Pharmacology Revue canadienne de physiologie et pharmacologie

\title{
Oxidative shift in tissue redox potential increases beat-to- beat variability of action potential duration
}

\begin{tabular}{|r|l|}
\hline Journal: & Canadian Journal of Physiology and Pharmacology \\
\hline Manuscript ID: & cjpp-2014-0531.R1 \\
\hline Manuscript Type: & Article \\
\hline Date Submitted by the Author: & n/a \\
\hline Complete List of Authors: & $\begin{array}{l}\text { Kistamas, Kornel; University of Debrecen, Department of Physiology } \\
\text { Hegyi, Bence; University of Debrecen, Department of Physiology } \\
\text { Vaczi, Krisztina; University of Debrecen, Department of Physiology } \\
\text { Horvath, Balazs; University of Debrecen, Department of Physiology } \\
\text { Banyasz, Tamas; University of Debrecen, Department of Physiology } \\
\text { Magyar, Janos; University of Debrecen, Department of Physiology } \\
\text { Szentandrassy, Norbert; University of Debrecen, Department of Physiology } \\
\text { Nanasi, Peter; University of Debrecen, Department of Physiology }\end{array}$ \\
\hline Keyword: & $\begin{array}{l}\text { redox potential, short term variability, action potential duration, canine } \\
\text { myocytes, beat-to-beat variability }\end{array}$ \\
\hline \multicolumn{2}{|c}{} \\
\hline
\end{tabular}


Oxidative shift in tissue redox potential increases beat-to-beat variability of action potential duration

Kornél Kistamás ${ }^{1}$, Bence Hegyi ${ }^{1}$, Krisztina Váczi ${ }^{1}$, Balázs Horváth ${ }^{1,2}$, Tamás Bányász ${ }^{1}$, János Magyar $^{1,4}$, Norbert Szentandrássy ${ }^{1,3}$, Péter P. Nánási ${ }^{1,3}$

${ }^{1}$ Department of Physiology, Faculty of Medicine, University of Debrecen, H-4012 Debrecen, P.O.Box 22, Hungary

${ }^{2}$ Faculty of Pharmacy, University of Debrecen, H-4012 Debrecen, P.O.Box 22, Hungary

${ }^{3}$ Department of Dental Physiology, Faculty of Dentistry, University of Debrecen, H-4012 Debrecen, P.O.Box 22, Hungary

${ }^{4}$ Division of Sport Physiology, Department of Physiology, Faculty of Medicine, University of Debrecen, H-4012 Debrecen, P.O.Box 22, Hungary

\section{Correspondence:}

P.P. Nánási at the address above

Phone: +36-52-255575

FAX: +36-52-255116

E-mail: nanasi.peter@med.unideb.hu 
Abstract: Profound changes of tissue redox potential occur in the heart under conditions of oxidative stress associated frequently with cardiac arrhythmias. Since beat-to-beat variability (short term variability, SV) of action potential duration (APD) is a good indicator of arrhythmia incidence, the aim of the present work was to study the influence of redox changes on SV in isolated canine ventricular cardiomyocytes using conventional microelectrode technique. The redox potential was shifted toward a reduced state using a reductive cocktail (containing dithiothreitol, glutathione and ascorbic acid) while oxidative changes were initiated by superfusion with $\mathrm{H}_{2} \mathrm{O}_{2}$. Redox effects were evaluated as changes in relative $S V$ determined by comparing SV changes to the concomitant APD changes. Exposure of myocytes to the reductive cocktail decreased SV significantly without any detectable effect on APD. Application of $\mathrm{H}_{2} \mathrm{O}_{2}$ increased both SV and APD, but the enhancement of SV was the greater, so relative SV increased. Longer exposure to $\mathrm{H}_{2} \mathrm{O}_{2}$ resulted in development of early afterdepolarizations accompanied by tremendously increased SV. Pretreatment with the reductive cocktail prevented both elevation of relative SV and the development of afterdepolarizations. The results suggest that the increased beat-to-beat variability during an oxidative stress contributes to generation of cardiac arrhythmias.

Keywords: redox potential, short term variability, action potential duration, beat-to-beat variability, canine myocytes.

\section{Introduction}

Beat-to-beat variability of action potential duration (short term variability, SV) is an intrinsic property of various mammalian cardiac preparations including the human heart (Hinterseer et al. 2009, Hinterseer et al. 2010, Tereshchenko et al. 2010). Since SV is considered as a good 
predictor of imminent arrhythmic events (Thomsen et al. 2004, Abi-Gerges et al. 2010, Jacobson et al. 2011) identification of factors involved in modulation of SV are important for basic research as well as for clinical practice. Involvement of several factors, such as stochastic gating of various ion channels (Lemay et al. 2011, Pueyo et al. 2011), intensity of cell-to-cell coupling (Zaniboni et al. 2000), morphology of the action potential (Heijman et al. 2013), stimulation frequency (Johnson et al. 2010) or changes in action potential duration (APD) and intracellular calcium concentration (Szentandrássy et al. 2014) in modulation of SV have been implicated so far.

Marked changes of redox potential in cardiac tissues occur under conditions of oxidative stress, such as in ischemia/reperfusion injury. On the other hand, increased incidence of cardiac arrhythmias is a common feature of these circumstances (Becker and Ambrosio 1987). Since beat-to-beat variability of action potential duration is believed to be a good indicator of arrhythmia incidence, it is logical to study the influence of changes in tissue redox potential on $\mathrm{SV}$ in isolated canine ventricular cardiomyocytes. This preparation was chosen because it is considered as a reasonably good model of human ventricular cells regarding their action potential morphology and kinetics of the underlying ion currents (Szabó et al. 2005, Szentandrássy et al. 2005), and also due to the significant amount of experimental data on SV accumulated already in dogs (see Szentandrássy et al. 2014 for references). The aim of this study was to study the effects of redox-shifts on relative $S V$ in canine ventricular myocytes in order to reveal their possible contribution to generation of cardiac arrhythmias.

\section{Materials and methods}

Isolation of single canine ventricular myocytes

Adult mongrel dogs of either sex were anaesthetized with intramuscular injections of $10 \mathrm{mg} / \mathrm{kg}$ 
ketamine hydrochloride (Calypsol, Richter Gedeon, Hungary) + 1 mg/kg xylazine hydrochloride (Sedaxylan, Eurovet Animal Health BV, The Netherlands) according to protocols approved by the local ethical committee (license $\mathrm{N}^{\mathrm{o}}$ : 18/2012/DEMÁB) in line with the ethical standards laid down in the Declaration of Helsinki in 1964 and its later amendments. Single myocytes were obtained by enzymatic dispersion using the segment perfusion technique, as described previously (Magyar et al. 2000). Briefly, the heart was quickly removed and washed in cold Krebs solution. A wedge-shaped section of the ventricular wall supplied by the left anterior descending coronary artery was dissected, cannulated and perfused with a nominally $\mathrm{Ca}^{2+}$-free Joklik solution (Minimum Essential Medium Eagle, Joklik Modification, Sigma-Aldrich Co. St. Louis, MO, USA) for 5 min. This was followed by 30 min perfusion with Joklik solution supplemented with $1 \mathrm{mg} / \mathrm{ml}$ collagenase (Type II, Worthington Biochemical Co., Lakewood, NJ, USA) and 0.2 \% bovine serum albumin (Fraction V., Sigma) containing $50 \mu \mathrm{M} \mathrm{Ca}^{2+}$. After gradually restoring the normal external $\mathrm{Ca}^{2+}$ concentration, the cells were stored in Minimum Essential Medium Eagle until use. Drugs were obtained from Sigma-Aldrich Co. (St. Louis, MO, USA).

\section{Recording and analysis of action potentials}

All experiments were performed at $37^{\circ} \mathrm{C}$, maintained by an electronic temperature controller (Cell MicroControls, Norfolk, VA, USA). The cells were sedimented in a plexiglass chamber of $1 \mathrm{ml}$ volume allowing continuous superfusion (at a rate of $2 \mathrm{ml} / \mathrm{min}$ ) with modified Krebs solution gassed with a mixture of $95 \% \mathrm{O}_{2}$ and $5 \% \mathrm{CO}_{2}$ at $\mathrm{pH}=7.4$. This solution contained (in $\mathrm{mM}) \mathrm{NaCl}, 128.3 ; \mathrm{NaHCO}_{3}, 21.4 ; \mathrm{KCl}, 4.0 ; \mathrm{CaCl}_{2}, 1.8 ; \mathrm{MgCl}_{2}, 0.42 ;$ and glucose 10. Transmembrane potentials were recorded using $3 \mathrm{M} \mathrm{KCl}$ filled sharp glass microelectrodes having tip resistance between 20 and $40 \mathrm{M} \Omega$. These electrodes were connected to the input of Multiclamp 700A or 700B amplifiers (Molecular Devices, Sunnyvale, CA, USA). The cells were 
paced through the recording electrode at steady cycle length of $1 \mathrm{~s}$ using 1-2 ms wide rectangular current pulses having amplitudes of twice the diastolic threshold. Since the cytosol was not dialyzed, time dependent changes in action potential morphology were negligible for the period of our experimental protocol lasting typically not longer than 15-20 min. Action potentials were digitized (at $200 \mathrm{kHz}$ using Digidata 1322A and 1440A, purchased from Molecular Devices, Sunnyvale, CA, USA) and stored for later analysis. Series of 50 consecutive action potentials were analyzed to estimate SV according to the following formula:

$\mathrm{SV}=\Sigma\left(\mid \mathrm{APD}_{\mathrm{n}+1}\right.$ minus $\left.\mathrm{APD}_{\mathrm{n}} \mid\right) /\left[\mathrm{n}_{\text {beats }} * \sqrt{ } 2\right]$

where SV is short term variability, $A P D_{n}$ and $A P D_{n+1}$ indicate the durations of the $n^{\text {th }}$ and $n+1^{\text {th }}$ APs, respectively, at $90 \%$ level of repolarization and $n_{\text {beats }}$ denotes the number of consecutive beats analyzed (Johnson et al. 2010, Szentandrássy et al. 2014). Changes in SV were typically presented as Poincaré plots where 50 consecutive APD values are plotted, each against the duration of the previous action potential. All control action potentials analyzed in the present study were normally driven ones, records taken in myocytes displaying early or delayed afterdepolarizations were excluded from evaluation.

\section{Statistics}

Results are expressed as mean \pm SEM values. Statistical significance of differences was evaluated using one-way ANOVA followed by Student's $t$-test. Differences were considered significant when $\mathrm{p}$ was less than 0.05 .

\section{Results}

As demonstrated in Figs. 1-2, exposure of myocytes to reductive environment had little effect on 
action potential morphology (Fig. 1.A,B), including the duration of action potentials measured at $90 \%$ level of repolarization (Fig. 2.A,D). In contrast, SV was significantly decreased by the reductive cocktail, containing $1 \mathrm{mM}$ DL-dithiothreitol, $1 \mathrm{mM}$ reduced L-glutathione and $1 \mathrm{mM}$ L-ascorbic acid (Fig. 1.C and Fig. 2.B,E). This effect of the reductive cocktail developed rapidly, the maximal reduction in SV was achieved within 6 min and SV failed to change afterwards. In order to offset the small and variable (statistically not significant) changes of APD developing in the reductive cocktail, relative $S V$ was determined by plotting a given SV value as a function of the corresponding APD (Fig. 2.C), and relative SV changes were estimated by plotting the changes in SV against the changes in APD (Fig. 2.F). The rationale of calculating relative SV values is that SV is strongly influenced by APD alone (Heijman et al. 2013, Szentandrássy et al. 2014). In order to eliminate the possible contribution of any concomitant change in APD, these data were compared to the exponential SV-APD relationship function obtained by manipulating APD using inward and outward current injections in absence of drug application - as reported earlier (Szentandrássy et al. 2014). The solid lines in panels C and F in Fig. 2 were obtained from such experiments (Szentandrássy et al. 2014). Data points appearing below the curve indicate reduction of relative $\mathrm{SV}$ in response of the reductive environment.

In contrast to reduction, oxidation caused profound changes in action potential morphology (Fig. 3). APD was progressively increased in the presence of the oxidant $10 \mu \mathrm{M}$ $\mathrm{H}_{2} \mathrm{O}_{2}$ (Fig. 3.B), and typically after 10-15 min of $\mathrm{H}_{2} \mathrm{O}_{2}$ superfusion early afterdepolarizations developed (Fig. 3.C-E). Since both APD and SV values were increased significantly by $\mathrm{H}_{2} \mathrm{O}_{2}$ (Fig. 4.A,D and Fig. 4.B,E, respectively), relative SV was determined. Relative SV was increased markedly by the oxidative shift in the redox potential, as demonstrated by the symbols located above the solid lines in panels $\mathrm{C}$ and F of Fig. 4. Elevation of SV was not accompanied by development of early afterdepolarizations within the initial 9 min exposure to $\mathrm{H}_{2} \mathrm{O}_{2}$. 
Finally, we aimed to show that these drastic effects of $\mathrm{H}_{2} \mathrm{O}_{2}$ superfusion are really consequences of redox potential changes. In the next series of experiments, therefore, $10 \mu \mathrm{M}$ $\mathrm{H}_{2} \mathrm{O}_{2}$ was applied in the presence of the reductive cocktail following a 5 min period of pretreatment. According to the results presented in Fig. 5, the elevation of relative SV, observed previously in $\mathrm{H}_{2} \mathrm{O}_{2}$, was fully prevented by the reductive environment having a sufficiently high redox buffering capacity (Fig. 5.C,F). This was true in spite of the fact that both SV and APD were increased by the $\mathrm{H}_{2} \mathrm{O}_{2}$ exposure (the former significantly, while the latter not significantly).

\section{Discussion}

In the present study we have shown that relative SV was decreased by applying a reductive environment, while shifting the redox potential to oxidative direction strongly increased relative SV. This was accompanied by development of early afterdepolarizations following the 10-15 min of $\mathrm{H}_{2} \mathrm{O}_{2}$ superfusion. These effects of $\mathrm{H}_{2} \mathrm{O}_{2}$ could be prevented by application of a reductive cocktail, containing DL-dithiothreitol, reduced L-glutathione and L-ascorbic acid - in $1 \mathrm{mM}$ concentration each. This composition was chosen to assure a sufficiently large redox buffering capacity, which might be necessary to safely reverse the effects of $\mathrm{H}_{2} \mathrm{O}_{2}$. This was critical, because we had to show that the strong effects of $\mathrm{H}_{2} \mathrm{O}_{2}$ were in fact consequences of an oxidative shift in the redox potential. Since similar changes are known to occur under conditions of oxidative stress (e.g. in the case of ischemia/reperfusion injury), it is plausible to assume that increases in beat-to-beat variability of APD, caused by an oxidative shift in the tissue redox potential, may contribute to the increased arrhythmia incidence observed under these pathological circumstances.

Regarding the possible underlying mechanisms, we can only speculate in the absence of relevant voltage clamp data. Effects of redox potential changes on cardiac ion currents display 
large variation according to the experimental conditions and the species studied. $\mathrm{I}_{\mathrm{Ca}}$ was markedly increased by oxidative challenges in the majority of cardiac cells, including canine (Hegyi et al. 2013), rabbit (Guo et al. 2000, Xie et al. 2009) and rat (Zhang et al. 2012) ventricular myocytes, similarly to HEK cells expressing the pore forming $\alpha_{1 \mathrm{C}}$ subunits of human L-type $\mathrm{Ca}^{2+}$ channels (Hudasek et al. 2004), while $\mathrm{I}_{\mathrm{Ca}}$ was decreased in guinea pig myocytes in response to oxidative stress (Cerbai et al. 1991, Goldhaber and Liu 1994). There was no change in $\mathrm{I}_{\mathrm{K} 1}$ amplitude in rabbit (Guo et al. 2000) and guinea pig (Cerbai et al. 1991), but the other inwardly rectifying $\mathrm{K}^{+}$current, the ATP-sensitive $\mathrm{K}^{+}$current $\left(\mathrm{I}_{\mathrm{K}-\mathrm{ATP}}\right)$, was enhanced by oxidation in the latter species (Goldhaber and Liu 1994, Ichinari et al. 1996). $\mathrm{I}_{\mathrm{Kr}}$ is likely to be enhanced by oxidation, since exposure of HERG channels - expressed in CHO cells - to $\mathrm{H}_{2} \mathrm{O}_{2}$ increased the amplitude of $\mathrm{I}_{\mathrm{Kr}}$ (Bérubé et al. 2001). These results, however, cannot explain the increased APD variability observed in the presence of $\mathrm{H}_{2} \mathrm{O}_{2}$, since both $\mathrm{I}_{\mathrm{Ca}}$ and $\mathrm{I}_{\mathrm{Kr}}$ were found to decrease $\mathrm{SV}$ in canine ventricular cells (Szentandrássy et al. 2014). Similar results were obtained in computer simulations (Heijman et al. 2013). $\mathrm{I}_{\mathrm{Ks}}$ was decreased by oxidative free radicals in guinea pig ventricular cells (Cerbai et al. 1991) and this current was also shown to decrease SV in canine myocytes and in silico models (Szentandrássy et al. 2014, Heijman et al. 2013). However, the limited contribution of $\mathrm{I}_{\mathrm{Ks}}$ to ventricular repolarization under baseline conditions in dogs (Varró et al. 2000, Volders et al. 2003) may question the role of $\mathrm{I}_{\mathrm{Ks}}$. Similarly, relative SV was not much affected by either $\mathrm{I}_{\mathrm{K} 1}$ or $\mathrm{I}_{\mathrm{K}-\mathrm{ATP}}$ in canine myocytes (Szentandrássy et al. 2014). The only ion current which may have a direct role in mediating the influence of redox changes on SV in dogs is $\mathrm{I}_{\mathrm{Na}}$. Amplitude of this current was increased by $\mathrm{H}_{2} \mathrm{O}_{2}$ in rabbit ventricular cells due to the removal of its fast inactivation (Xie et al. 2009), and $\mathrm{I}_{\mathrm{Na}}$ was shown to increase $\mathrm{SV}$ in dogs (Szentandrássy et al. 2014) as well as in silico (Heijman et al. 2013). However, in absence of relevant voltage clamp data obtained in canine ventricular cells, the possible role of $\mathrm{I}_{\mathrm{Na}}$ remains 
to be supported experimentally.

Importantly, elevation of $\left[\mathrm{Na}^{+}\right]_{\mathrm{i}}$ is known to be converted to intracellular $\mathrm{Ca}^{2+}$ accumulation. Indeed, accumulation of cytosolic $\mathrm{Ca}^{2+}$ was observed with $\mathrm{H}_{2} \mathrm{O}_{2}$ in rabbit ventricular cells (Goldhaber 1996, Xie et al. 2009), which shift has been reported to increase the beat-to-beat variability of APD (Johnson et al. 2013, Szentandrássy et al. 2014). The casual role of the elevated $\left[\mathrm{Ca}^{2+}\right]_{\mathrm{i}}$ in the increased variability is further supported by the documented actions of oxidative shifts resulting in elevation of $\left[\mathrm{Ca}^{2+}\right]_{\mathrm{i}}$. Several redox sensitive mechanisms were shown to increase $\left[\mathrm{Ca}^{2+}\right]_{\mathrm{i}}$ during oxidative stress, including the higher open probability of ryanodine receptor (Anzai et al. 2000, Hidalgo et al. 2004, Zissimopoulos and Lai 2006), the increased amplitude of $\mathrm{I}_{\mathrm{Ca}}$ (Guo et al. 2000, Hudasek et al. 2004, Hidalgo et al. 2004, Xie et al. 2009, Zhang et al. 2012, Hegyi et al. 2013) accompanied by an enhanced $\mathrm{Na}^{+} / \mathrm{Ca}^{2+}$ exchange current (Goldhaber 1996). All these changes are known to increase beat-to-beat variability and evoke afterdepolarizations and therefore might account for the actions of the oxidative environment on beat-to-beat variability. Direct evidence for this mechanism has recently been provided by Bonilla et al. (2013) reporting that peroxynitrite accumulation increased SV in canine ventricular, but not in atrial, cardiomyocytes. Further investigation is required to reveal the reason for this interesting atrio-ventricular difference.

In summary, the results of the present study indicate that relative $S V$ is increased by shifting the redox potential towards oxidative direction, while a reductive cocktail had an opposite effect, suggesting that the increased beat-to-beat variability during an oxidative stress may play an important role in generation of cardiac arrhythmias under these pathological conditions.

\section{Acknowledgements}


Financial support was provided by grants from the Hungarian Scientific Research Fund (OTKAK100151, OTKA-K109736, OTKA-K101196, OTKA-PD101171 and OTKA-NK104331). Further support was obtained from the Hungarian Government and the European Community (TAMOP-4.2.2.A-11/1/KONV-2012-0045 research project) and National Excellence Program (TAMOP-4.2.4.A/2-11/1-2012-0001) to BH and Bolyai János Research Scholarship to NS. The authors thank Miss Éva Sági for excellent technical assistance. The authors declare that they have no conflict of interest. 


\section{References}

Abi-Gerges, N., Valentin, J.P., Pollard, C.E. 2010. Dog left ventricular midmyocardial myocytes for assessment of drug-induced delayed repolarization: short-term variability and proarrhythmic potential. Br. J. Pharmacol. 159: 77-92.

Anzai, K., Ogawa, K., Ozawa, T., Yamamoto, H. 2000. Oxidative modification of ion channel activity of ryanodine receptor. Antioxid. Redox Signal. 2: 35-40.

Becker, L.C., Ambrosio, G. 1987. Myocardial consequences of reperfusion. Prog. Cardiovasc. Dis. 30: $23-44$.

Bérubé, J., Caouette, D., Daleau, P. 2001. Hydogen peroxid modifies the kinetics of HERG channel expressed in a mammalian cell line. J. Pharmacol. Exp. Ther. 297: 96-102.

Bonilla, I.M., Sridhar, A., Nishijima, Y., Györke, S., Cardounel, A.J., Carnes, C.A. 2013. Differencial effects of the peroxynitrite donor, SIN-1, on atrial and ventricular myocyte electrophysiology. J. Cardiovasc. Pharmacol. 61: 401-407.

Cerbai, E., Ambrosio, G., Porciatti, F., Chiariello, M., Giotti, A., Mugelli, A. 1991. Cellular electrophysiological basis for oxygen radical-induced arrhythmias. A patch-clamp study in guinea pig ventricular myocytes. Circulation 84: 1773-1782.

Goldhaber, J.I. 1996. Free radicals enhance $\mathrm{Na}^{+} / \mathrm{Ca}^{2+}$ exchange in ventricular myocytes. Am. J. Physiol. 271: H823-H833.

Goldhaber, J.I., Liu, E. 1994. Excitation-contraction coupling in single guinea-pig ventricular myocytes exposed to hydrogen peroxide. J. Physiol. (Lond.) 477: 135-147.

Guo, J., Giles, W.R., Ward, C.A. 2000. Effect of hydrogen peroxide on the membrane currents in sinoatrial node cells from rabbit heart. Am. J. Physiol. Heart Circ. Physiol. 279: H992-H999.

Hegyi, B., Komáromi, I., Kistamás, K., Ruzsnavszky, F., Váczi, K., Horváth, B., Magyar, J., 
Bányász, T., Nánási, P.P., Szentandrássy, N. 2013. Tetrodotoxin blockade on canine cardiac Ltype $\mathrm{Ca}^{2+}$ channels depends on $\mathrm{pH}$ and redox potential. Marine Drugs 11: 2140-2153.

Heijman, J., Zaza, A., Johnson, D.M., Rudy, Y., Peeters, R.L.M., Volders, P.G.A., Westra, R.L. 2013. Determinants of beat-to-beat variability of repolarization duration in the canine ventricular myocyte: a computational analysis. PLOS Comput. Biol. 9: e1003202.

Hidalgo, C., Bull, R., Behrens, M.I., Donoso, P. 2004. Redox regulation of RyR-mediated Ca ${ }^{2+}$ release in muscle and neurons. Biol. Res. 37: 539-552.

Hinterseer, M., Beckmann, BM., Thomsen, M.B., Pfeufer, A., Dalla Pozza, R., Loeff, M., Netz, H., Steinbeck, G., Vos, M.A., Kääb, S. 2009. Relation of increased short-term variability of QT interval to congenital long-QT syndrome. Am. J. Cardiol. 103: 1244-1248.

Hinterseer, M., Beckmann, B.M., Thomsen, M.B., Pfeufer, A., Ulbrich, M., Sinner, M.F., Perz, S., Wichmann, H.E., Lengyel, C., Schimpf, R., Maier, S.K., Varró, A., Vos, M.A., Steinbeck, G., Kääb, S. 2010. Usefulness of short-term variability of QT intervals as a predictor for electrical remodeling and proarrhythmia in patients with nonischemic heart failure. Am. J. Cardiol. 106: 216-220.

Hudasek, K., Brown, S.T., Fearon, I.M. 2004. $\mathrm{H}_{2} \mathrm{O}_{2}$ regulates recombinant $\mathrm{Ca}^{2+}$ channel $\alpha_{1 \mathrm{C}}$ subunits but does not mediate their sensitivity to acute hypoxia. Biochem. Biophys. Res. Commun. 318: 135-141.

Ichinari, K., Kakei, M., Matsuoka, T., Nakashima, H., Tanaka, H. 1996. Direct activation of the ATP-sensitive potassium channel by oxygen free radicals in guinea-pig ventricular cells: its potentiation by MgADP. J. Mol. Cell. Cardiol. 28: 1867-1877.

Jacobson, I., Carlsson, L., Duker, G. 2011. Beat-by-beat QT interval variability, but not QT prolongation per se, predicts drug-induced torsades de pointes in the anaesthetised methoxaminesensitized rabbit. J. Pharmacol. Toxicol. Methods 63: 40-46. 
Johnson, D.M., Heijman, J., Pollard, C.E., Valentin, J.P., Crijns, H.J., Abi-Gerges, N., Volders, P.G. 2010. $\mathrm{I}_{\mathrm{Ks}}$ restricts excessive beat-to-beat variability of repolarization during beta-adrenergic receptor stimulation. J. Mol. Cell. Cardiol. 48: 122-130.

Johnson, D.M., Heijman, J., Bode, E.F., Greensmith, D.J., Van der Linde, H., Abi-Gerges, N., Eisner, D.A., Trafford, A.W., Volders, P.G. 2013. Diastolic spontaneous calcium release from the sarcoplasmic reticulum increases beat-to-beat variability of repolarization in canine ventricular myocytes after $\beta$-adrenergic stimulation. Circ. Res. 112: 246-256.

Lemay, M., de Lange, E., Kucera, J.P. 2011. Effects of stochastic channel gating and distribution on the cardiac action potential. J. Theor. Biol. 281: 84-96.

Magyar, J., Bányász, T., Szigligeti, P., Körtvély, Á., Jednákovits, A., Nánási, P.P. 2000. Electrophysiological effects of bimoclomol in canine ventricular myocytes. Naunyn Schmiedeberg's Arch. Pharmacol. 361:303-310.

Pueyo, E., Corrias, A., Virág, L., Jost, N., Szél, T., Varró, A., Szentandrássy, N., Nánási, P.P., Burrage, K., Rodríguez, B. 2011. A multiscale investigation of repolarization variability and its role in cardiac arrhythmogenesis. Biophys. J. 101: 2892-2902.

Szabó, G., Szentandrássy, N., Bíró, T., Tóth, B.I., Czifra, G., Magyar, J., Bányász, T., Varró, A., Kovács, L., Nánási, P.P. 2005. Asymmetrical distribution of ion channels in canine and human left ventricular wall: epicardium versus midmyocardium. Pflugers Arch. 450: 307-316.

Szentandrássy, N., Bányász, T., Bíró, T., Szabó, G., Tóth, B.I., Magyar, J., Lázár, J., Varró, A., Kovács, L., Nánási, P.P. 2005. Apico-basal inhomogeneity in distribution of ion channels in canine and human ventricular myocardium. Cardiovasc. Res. 65: 851-860.

Szentandrássy, N., Kistamás, K., Hegyi, B., Horváth, B., Ruzsnavszky, F., Váczi, K., Magyar, J., Bányász, T., Varró, A., Nánási, P.P. 2014. Contribution of ion currents to beat-to-beat variability of action potential duration in canine ventricular myocytes. Pflügers Arch. Epub ahead to print. 
DOI: $10.1007 / \mathrm{s} 00424-014-1581-4$.

Tereshchenko, L.G., Han, L., Cheng, A., Marine, J.E., Spragg, D.D., Sinha, S., Dalal, D., Calkins, H., Tomaselli, G.F., Berger, R.D. 2010. Beat-to-beat three-dimensional ECG variability predicts ventricular arrhythmia in ICD recipients. Heart Rhythm 7: 1606-1613.

Thomsen, M.B., Verduyn, S.C., Stengl, M., Beekman, J.D., de Pater, G., van Opstal, J., Volders, P.G., Vos, M.A. 2004. Increased short-term variability of repolarization predicts d-sotalolinduced torsades de pointes in dogs. Circulation 110: 2453-2459.

Varró, A., Baláti, B., Iost, N., Takács, J., Virág, L., Lathrop, D.A., Lengyel, C., Tálosi, L., Papp, J.G. 2000. The role of the delayed rectifier component $\mathrm{I}_{\mathrm{Ks}}$ in dog ventricular muscle and Purkinje fibre repolarization. J. Physiol. (Lond.) 523: 67-81.

Volders, P.G.A., Stengl, M., van Opstal, J.M., Gerlach, U., Spatjens, R.L.H.M.G., Beekman, J.D.M., Sipido, K.R., Vos, M.A. 2003. Probing the contribution of $\mathrm{I}_{\mathrm{Ks}}$ to canine ventricular repolarization: key role for $\beta$-adrenergic receptor stimulation. Circulation 107, 2753-2760.

Xie, L.H., Chen, F., Karagueuzian, H.S., Weiss, J.N. 2009. Oxidative-stress-induced afterdepolarizations and calmodulin kinase II signaling. Circ. Res. 104: 79-86.

Zaniboni, M., Pollard, A.E., Yang, L., Spitzer, K.W. 2000. Beat-to-beat repolarization variability in ventricular myocytes and its suppression by electrical coupling. Am. J. Physiol. Heart. Circ. Physiol. 278: H677-H687.

Zhang, R., Sun, Y., Tsai, H., Tang, C., Jin, H., Du, J. 2012. Hydrogen sulfide inhibits L-type calcium currents depending upon the protein sulfhydryl state in rat cardiomyocytes. PLoS One 7, e37073.

Zissimopoulos, S., Lai, F.A. 2006. Redox regulation of the ryanodine receptor/calcium release channel. Biochem. Soc. Trans. 34: 919-921. 


\section{Figure legends}

Fig. 1. Effect of reductive cocktail on isolated canine ventricular action potentials. Superimposed sets of 50 consecutive action potentials in control (A) and after exposure to a reductive cocktail, containing DL-dithiothreitol, reduced L-glutathione and $1 \mathrm{mM}$ L-ascorbic acid, each in $1 \mathrm{mM}$ concentration (B). Results were also displayed using a Poincaré plot (C).

Fig. 2. Time-dependent effects of superfusion with reductive cocktail on action potential duration $(\mathbf{A}, \mathbf{D})$ and beat-to-beat variability $(\mathbf{B}, \mathbf{E})$. In order to visualize relative $\mathrm{SV}$, SV was plotted as a function of APD in panel $\mathbf{C}$, while in $\mathbf{F}$ the corresponding changes, induced by the reductive cocktail, are shown. Solid curves in panels $\mathbf{C}$ and $\mathbf{F}$ indicate those SV-APD and $\triangle \mathrm{SV}$ $\triangle \mathrm{APD}$ relationships, respectively, which were obtained earlier when APD was shortened or lengthened within a wide range using outward and inward current pulses, injected throughout the entire duration of the action potentials. (Data from Szentandrássy et al. 2014). Symbols and bars represent mean \pm SEM values obtained in 12 myocytes, asterisks denote significant differences from the control $(\mathrm{p}<0.05)$.

Fig. 3. Representative experiment demonstrating the effect of oxidative environment on isolated canine ventricular action potentials. Superimposed sets of 50 consecutive action potentials in control (A) and after exposure to $10 \mu \mathrm{M} \mathrm{H}_{2} \mathrm{O}_{2}$ for 9 (B) and $12 \mathrm{~min}$ (C). D: Poincaré diagram showing the changes in APD and its beat-to-beat variation. E: Record displaying a 30 min period of the experiment, including early afterdepolarizations developing in the presence of longer exposures to $\mathrm{H}_{2} \mathrm{O}_{2}$. 
Fig. 4. Time-dependent effects of $10 \mu \mathrm{M} \mathrm{H}_{2} \mathrm{O}_{2}$ on action potential duration (A, D) and beat-tobeat variability $(\mathbf{B}, \mathbf{E})$. Average relative SV values and their changes, respectively, evoked by $\mathrm{H}_{2} \mathrm{O}_{2}$ are presented in panels $\mathbf{C}$ and $\mathbf{F}$. The solid curves were derived identically as in the case of Fig. 2, according to Szentandrássy et al. 2014. Symbols and bars are means \pm SEM obtained in 10 cells, asterisks denote significant differences from control $(\mathrm{p}<0.05)$.

Fig. 5. Average results demonstrating that $5 \mathrm{~min}$ pre-treatment with the reductive cocktail was able to prevent the consequences of a subsequent exposure to $10 \mu \mathrm{M} \mathrm{H}_{2} \mathrm{O}_{2}$. Effects of $\mathrm{H}_{2} \mathrm{O}_{2}$ on $\operatorname{APD}(\mathbf{A}, \mathbf{D})$ and SV $(\mathbf{B}, \mathbf{C})$ in the presence of reductive cocktail. Symbols and bars indicate means \pm SEM obtained in 6 myocytes, asterisks here denote significant changes $(\mathrm{p}<0.05)$ from data obtained in the reductive cocktail before exposure to $\mathrm{H}_{2} \mathrm{O}_{2}$. As demonstrated in panels $\mathbf{C}$ and $\mathbf{F}$, no change in relative SV was observed under these conditions. 

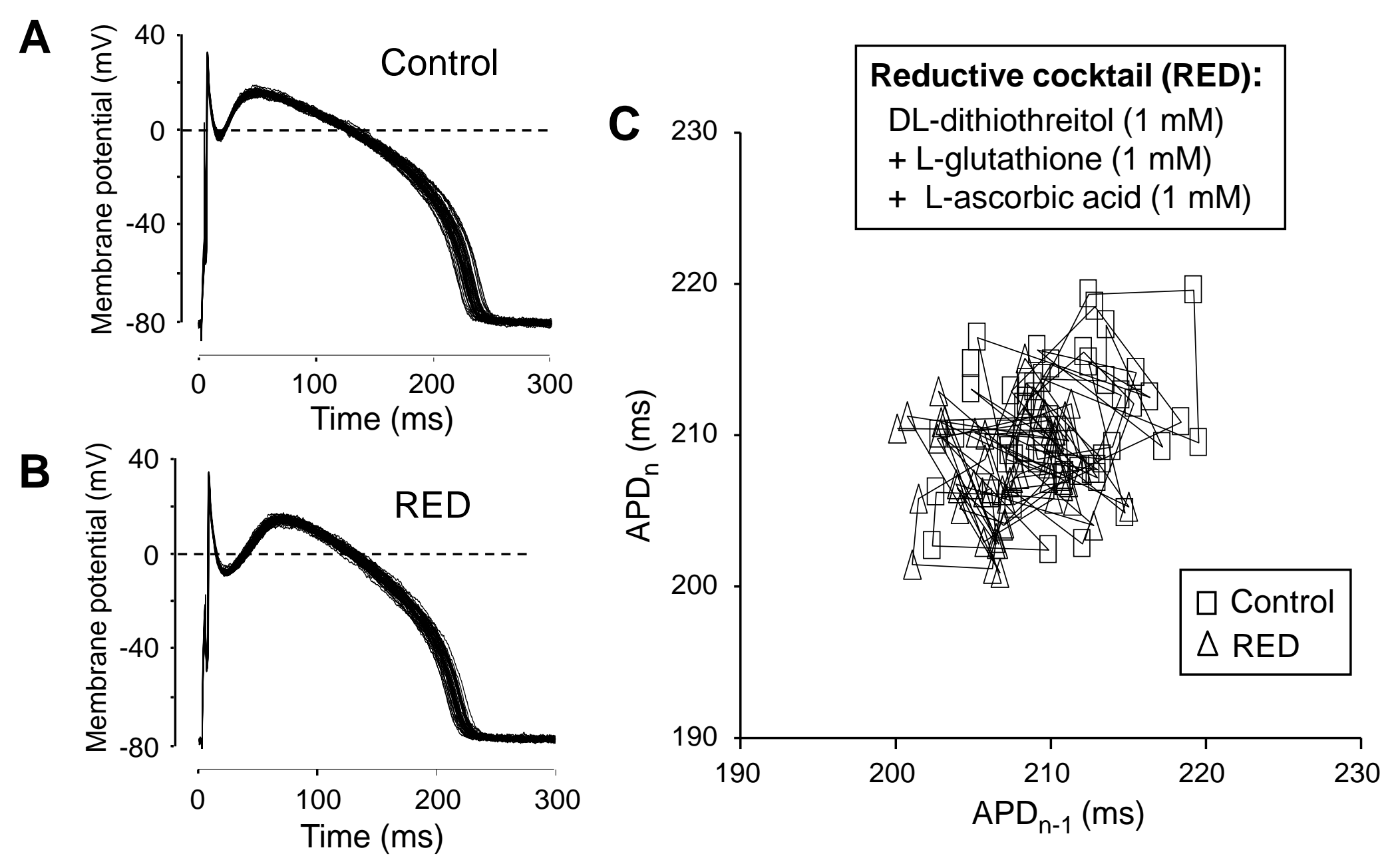

Fijugriptcentral.com/cjpp-pubs 

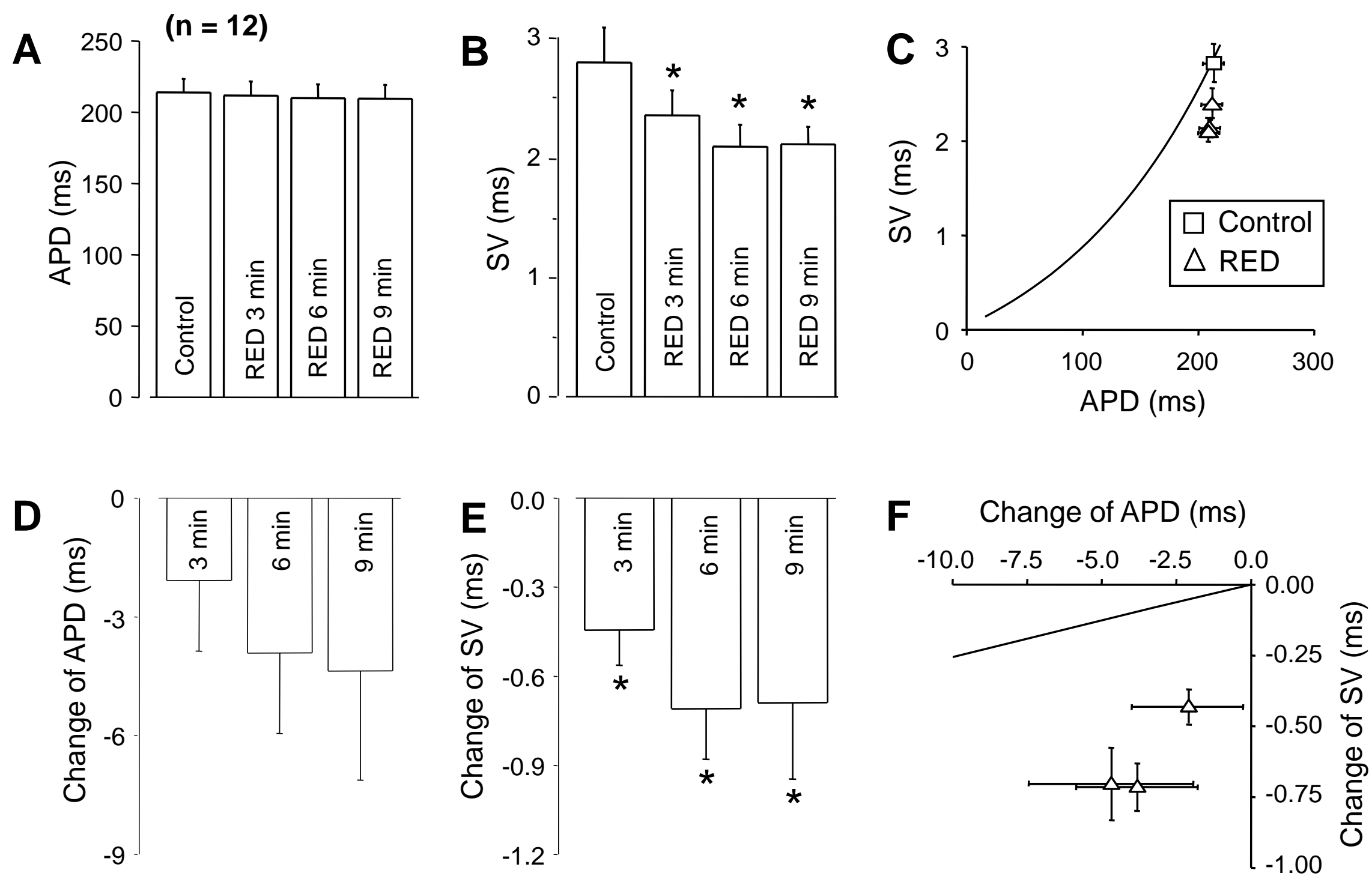

Fig ${ }_{\text {marifentral.com/cjpp-pubs }}$ 
Page 19 of 21
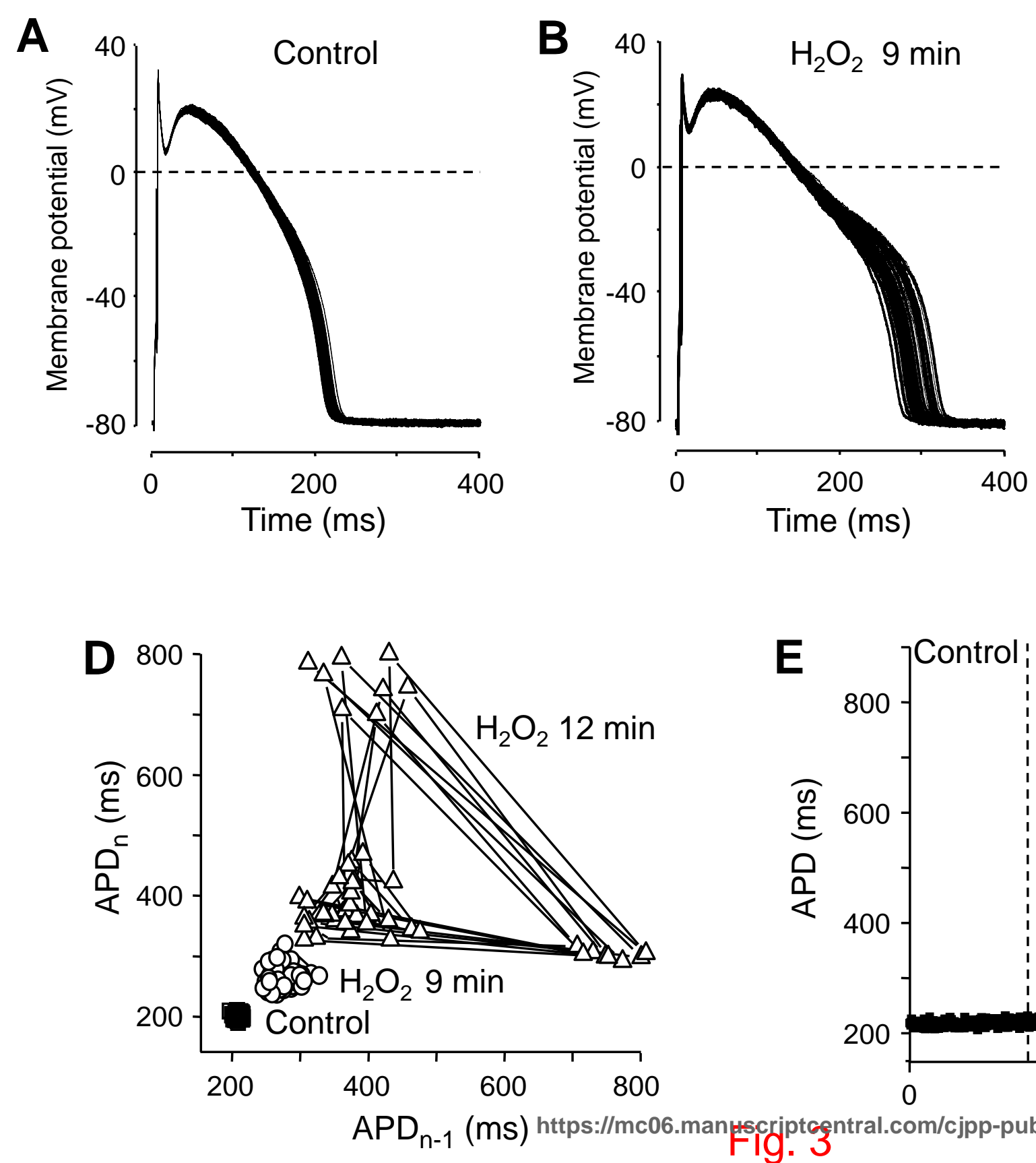

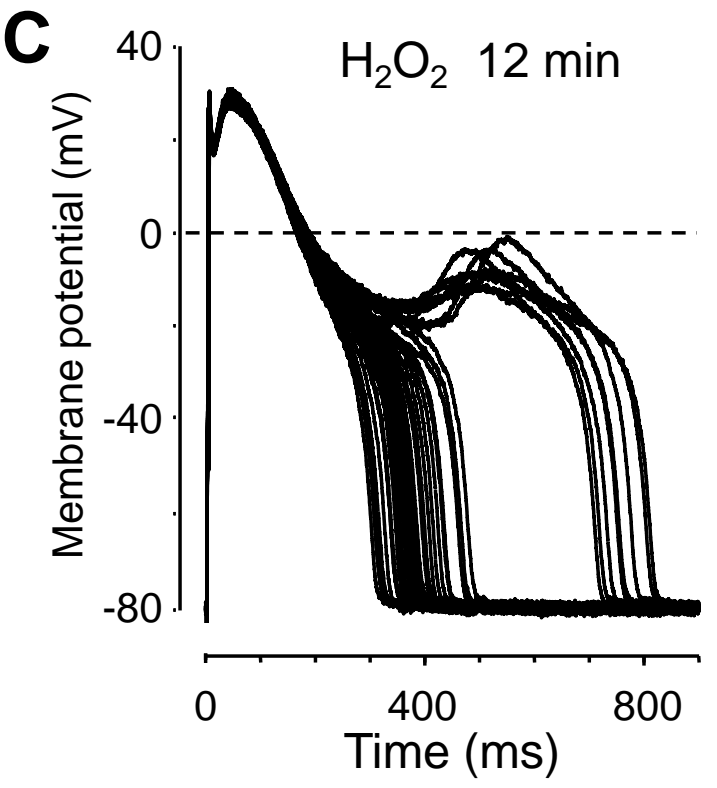

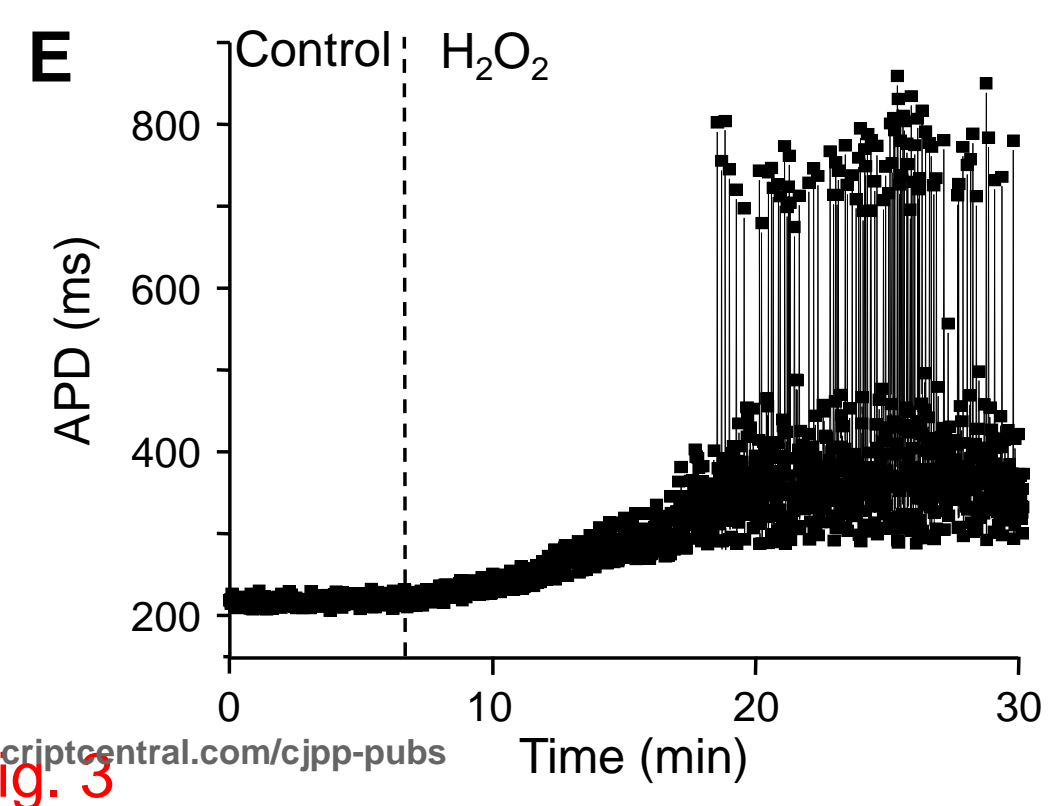



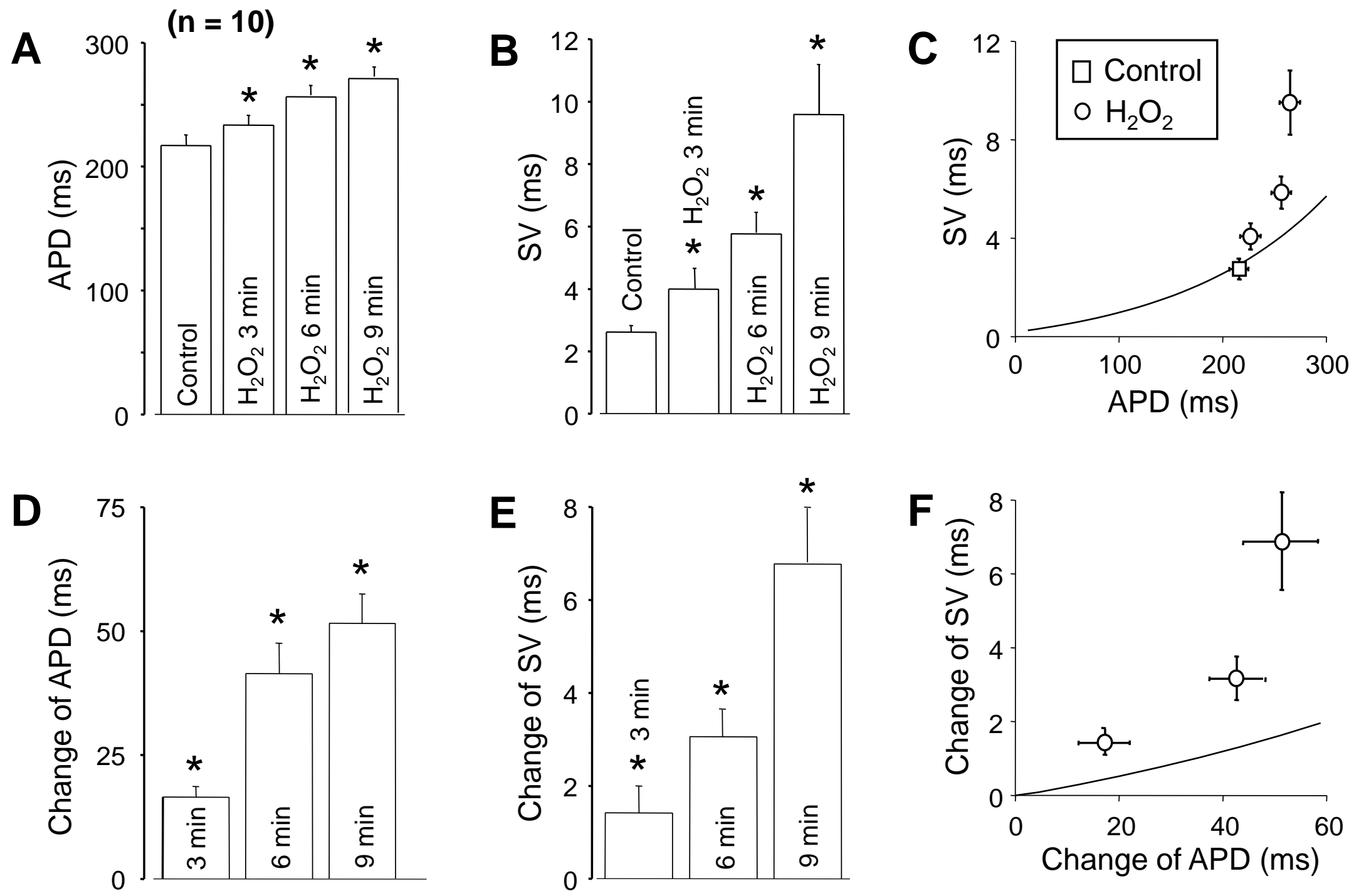

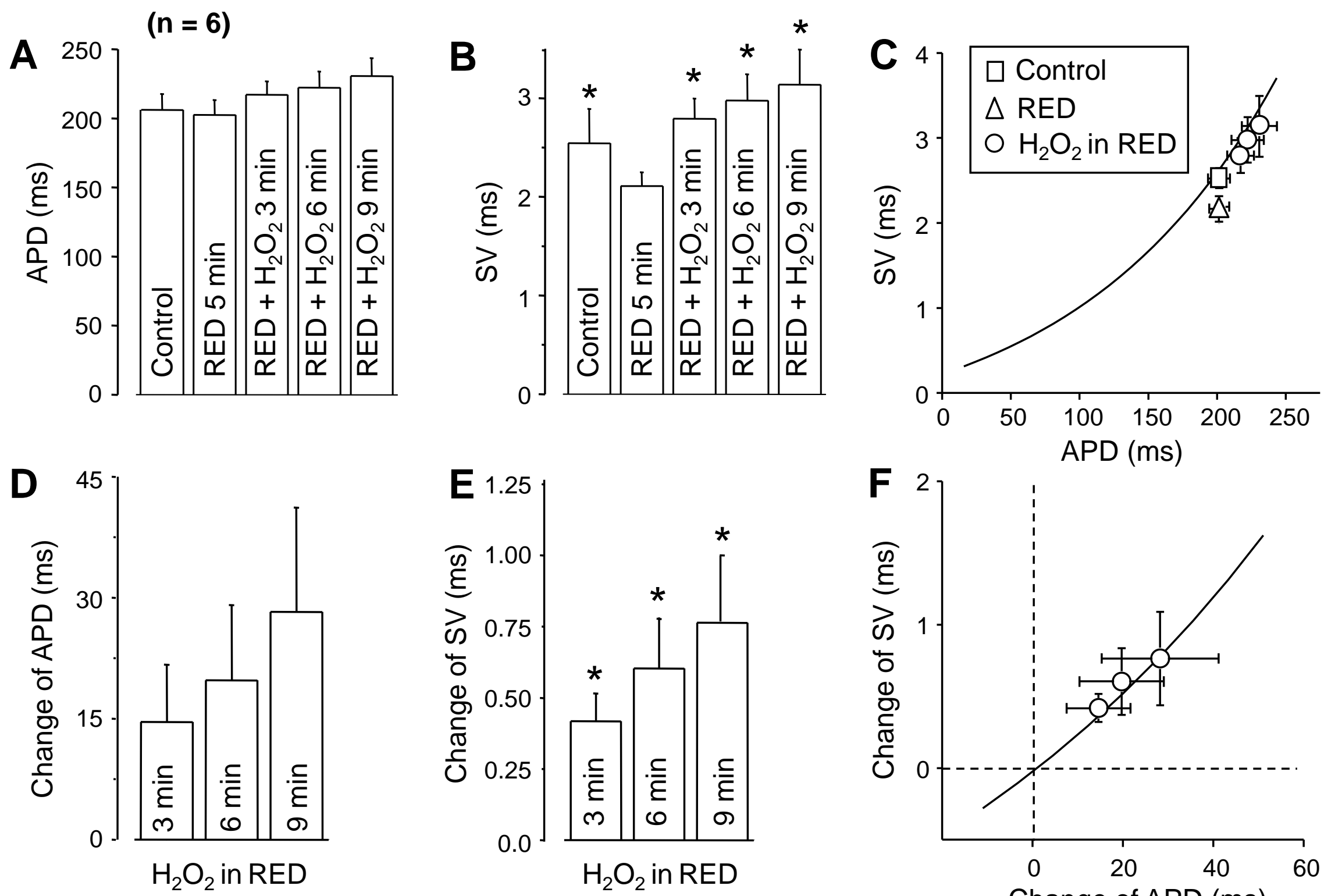

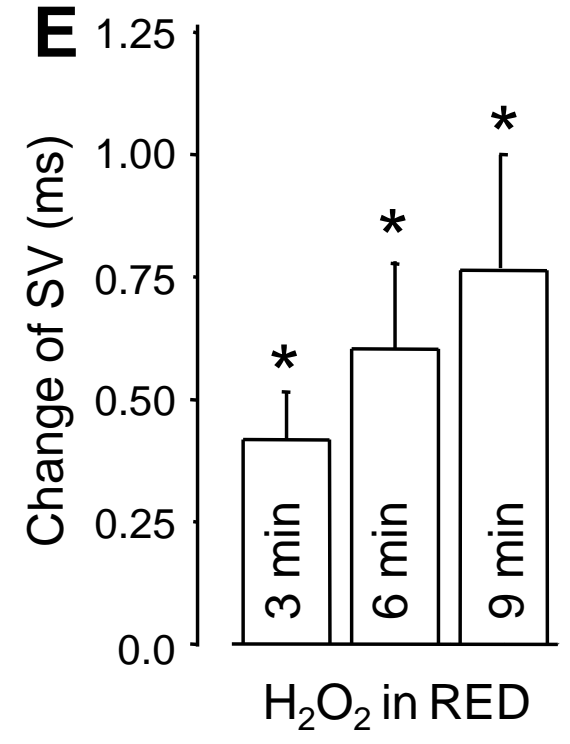

https://mc06.manuscriptcentral.com/cjpp-pubs Fig. 5. 